\title{
TRIPLE NEGATIVE BREAST CANCER. CURRENT THERAPEUTIC OPTIONS IN THE NEOADJUVANT SETTING
}

\author{
Nicolae Bacalbasa ${ }^{1}$, Olivia Ionescu ${ }^{2}$ \\ I "Carol Davila" University of Medicine and Pharmacy, Bucharest \\ 2 "Bucur” Maternity, Bucharest
}

\begin{abstract}
At present, breast cancer $(\mathrm{BC})$ has the highest incidence among the most common forms of cancer in women, almost one million women being diagnosed annually worldwide. However, in recent years BC has registered a low mortality rate mainly in developed, high-income countries. The triple negative breast cancer (TNBC) is a subtype of BC which is characterized by the absence of protein expression of the two hormone receptors-estrogen $(\mathrm{ER})$ and progesterone $(\mathrm{PgR})$ after immunohistochemistry $(\mathrm{IHC})$ analysis and the lack of overexpression of the human epidermal growth factor receptor 2 (HER2) after IHC or in situ fluorescence hybridization technique. More than 170,000 of women are currently diagnosed with TNBC (ER-/PR-/HER2) representing $12-20 \%$ of all $\mathrm{BC}$.

TNBCs are recognized to have a poor prognosis which translates into a relative low disease-free survival rate for women who receive either neoadjuvant or adjuvant chemotherapy as well as a low progression-free survival rate for women who develop distant metastases. Furthermore, biologically, they are much more aggressive than the other types of BC and, owing to their triple "negativity", a targeted therapy with anti-hormon agents or transtuzumab (anti HER2) cannot be utilized.

The aim of this paper is to make a review of the current scientific evidence with regard to the new chemotherapeutic agents used in the neoadjuvant setting as well as the role of surgical treatment in women diagnosed with TNBC.
\end{abstract}

Keywords: breast cancer, triple negative, chemotherapy, neoadjuvant

\author{
Abbreviations: \\ $\mathrm{BC}=$ breast cancer; \\ TNBC = triple negative breast cancer; \\ $\mathrm{ER}=$ estrogen receptor; \\ $\mathrm{PgR}=$ progesterone receptor; \\ HER2 = human epidermal growth factor;
}

\section{INTRODUCTION}

Worldwide, breast cancer (BC) is the most common cancer among women, $23 \%$ of all new diagnosed cancers being BC. Of all these BCs, triple negative breast cancer (TNBC), defined as the lack of estrogen, progesterone and human epidermal growth factor 2 receptors, represents $12 \%-20 \%$ or 170,000 cases. (1)

TNBC comprises several molecular subtypes which require different treatment options and are
OS = overall survival;

DFS = disease free survival;

$\mathrm{BCS}=$ breast conservative surgery;

$\mathrm{LN}=$ lymph node;

GBG = german breast group.

more or less sensitive to chemotherapy or biological agents. Among these we can mention: the basallike (BL1 and BL2) which represents $75 \%$ of all the TNBC, the immunomodulatory (IM), the mesenchymal (M), the mesenchymal stem-like (MSL), and the luminal androgen receptor (LAR) subtype (2). Moreover, studies have revealed a correlation between BRCA1 mutations and the predisposition to TNBC. It seems that the presence of a germ-line BRCA1 mutations predisposes to TNBC especially the basal-like subtype. (3) 
TNBC is encountered in almost $39 \%$ of all AfroAmerican women in premenopausal age, a percentage which is 3-fold higher than that of TNBC in white women as presented in 2006 in San Antonio Breast Cancer Symposium. $(4,5)$ Females who develop TNBC are younger than those with $\mathrm{ER}+/$ Her2 + or with ER+/Her2-, live in low socioeconomic conditions and generally present an aggressive clinical course with low OS and DFS rates in the adjuvant and neoadjuvant setting. (6) Dent and his coworkers (7) presented their study at the 2015 American Society of Clinical Oncology (ASCO) Meeting and showed that of 10,000 women diagnosed with TNBC in the USA in 2010 and 2011, $34 \%$ were at stage I, $42 \%$ at stage II, $15 \%$ at stage III, and $6 \%$ at stage IV. The 24-month OS rates were $97 \%, 93 \%, 71 \%$, and $27 \%$, respectively, while the median OS in the metastatic setting was 13 months.

Besides the high probability of coexistence of the BRCA1 mutation, TNBC have a generally weak prognosis with a high risk of early relapse after completion after the adjuvant treatment, especially during the first and third year. They grow quickly and their tissular density resemble that of the normal tissue. Moreover, there is an inverse relationship between the size of the tumor and the presence of lymph node (LN) metastases. The most frequent location of metastases are those located in the brain. Once diagnosed the metastatic disease, a rapid progression of the disease has been observed, most of the deaths occurring in the first 5 years after metastasis onset. $(8,9)$

With regard to the treatment possibilities of TNBC, as antihormons and transtuzumab cannot be used, the only possible options remain surgery and chemotherapy given either individually or in combination. In this review, our purpose is to present the current therapeutic possibilities for women diagnosed with TNBC.

\section{NEOADJUVANT CHEMOTHERAPY}

The specific chemotherapeutic agents in TNBC patients have not been precisely defined, there is evidence that TNBC can be approached with chemotherapeutic drugs such as combination of platinum-based agents and taxanes which target DNA repair, taxanes against oncoprotein P-53 and anthracyclines against cell proliferation. Currently, both in early and in the advanced disease, dose dense or metronomic polychemotherapy are the most effective options. (10)
Once that TNBC has been demonstrated to be strongly associated with presence of BRCA1 mutation and impaired DNA repair, platinum-based agents as well as etoposide and bleomycin can be currently administered. (11) The role of taxanes in TNBC has been proved to be essential while BCs which present p-53 mutations have been demonstrated to be resistant to anthracycline-based agents. (12)

Studies have showed that performing neoadjuvant chemotherapy (chemotherapy before operation) in TNBC patients results in significant benefits benefits on the OS rate and a better clinical and pathological complete response rate especially in TNBC with high expression of Ki-67. Moreover, the pathological complete response is considered to be an important parameter which is strongly correlated with the survival outcome. (13)

\section{Anthracycline and taxanes}

The retrospective study of Wang and coworkers (14) performed in 2009 on women with TNBC who received neoadjuvant chemotherapy with anthracycline and taxanes a significantly high pathological complete response has been observed among women with TNBC (38\%) compared to non- TNBC $(12 \%)$ as well as a better DFS rate. (14) Another trial NSABP B-27 showed that the use preoperative standard neoadjuvant regimen with anthracycline + cyclophosphamide and docetaxel followed by surgery also results in a significant increased pathological complete response - $26.1 \%$ compared to $12.1 \%$ when only anthracycline and cyclophosphamide have preoperatively been used. However, these preoperative regimen (four cycles of anthracycline and cyclophosphamide followed by four cycles of docetaxel) did not have any significant impact on the DFS or OS rate. (15)

\section{Platinum agents and BRCA1 mutation}

The use of cisplatin in the treatment of TNBC has progressively gained interest as it has been showed that TNBC has a higher incidents among women with BRCA1 gene mutation. The histologic similarities between TNBC and tumor cell lines deficient in BRCA1 has been demonstrated in several clinical trials as well as the high sensitivity of BRCA 1 mutated BC to DNA cross-linking agents such as cisplatin. (16)

The effectiveness of cisplatin has been investigated by Garber and coworkers (17) who preoperatively administered four cycles of cisplatin in wom- 
en with stage II or III TNBC and BRCA1 mutation. At the completion of chemotherapy, $22 \%$ of women achieved a pathological clinical response while $36 \%$ presented a complete or near-complete response. Moreover, remarkable results have also been obtained when cisplatin has been used with other agents (e.g. paclitaxel and epirubicin) - a pathological clinical response of $65 \%$. After surgical treatment and adjuvant chemotherapy with 4 cycles of cyclophosphamide, methotrexate, and flurouracil the reported 3-year and 5-year DFS were $97 \%$ respectively $90 \%$. (18)

\section{Antimetabolits}

The studies regarding the utility of antimetabolits in TNBC refer to the preoperative addition of capecitabine to docetaxel wither sequentially or combined. One of these studies has been conducted by Zelnak and coworokers (19) who obtained a pathologically clinical response of $8 \%$ when the neoadjuvant 4 cycles of docetaxel have been followed by 4 cycles of the antimetabolite capecitabine and $11.5 \%$ when 8 cycles of concurrent capeciabine and docetaxel have been administered. In the same way, Albain et al (20) showed that the preoperative use of the antimetabolite gemcitabine added to paclitaxel resulted in an increased response rate (40.8\%) and median survival (18.5 months).

In spite of these encouraging results we should not omit to take into consideration the treatment's toxicity when antimetabolits are added to taxanebased regimens or combined anthracyclines and taxanes. This is why, novel therapeutic agents which can target specific molecular factors are required in order to increase the response rate to the chemotherapeutic regimen and decrease the associated side effects.

\section{Antiangiogenic agents}

The most challenging issue when it comes to the use of antiangiogenic agents refer to the use of bevacizumab in the neoadjuvant setting. Although, the GeparQuinto study (21) performed in 2013 by the German Breast Group 44 did not show a significant improvement of the pathological clinical response of women who received neoadjuvantly 4 cycles of epirubicin and cyclophosphamide followed by 4 cycles of docetaxel plus bevacizumab (anti-vascular endothelial growth factor anti-VEGF) compared to those who were not administered bevacizumab (pathological clinical response $17.5 \%$ vs. $15 \%$ ), however this improvement is encouraging and shows that the use of bevacizumab may result in a higher probability of increased pathological clinical response.

Following the same direction as the GBG 44, the ASCO annual meeting from 2011 (22) conducted an analysis which showed the pathological clinical response rates were higher in the case of TNBC patients with positive LNs who received bevacizumab added to epirubicine/cyclophosphamide and taxane $(36,4 \%)$ compared to that of women who were not added bevacizumab (28\%).

The results of adding platinum and bevacizum$\mathrm{ab}$ to anthracycline-taxane based regimens are currently investigated in the CALGB study 40603 (23) and the expected results are awaited to impose a novel neoadjuvant regimen which may improve the long-term outcomes of TNBC patients.

\section{Antineoplastic agents}

The use of the antineoplastic agents is based on their ability to stabilize microtubules and cause cell cycle arrest and apoptosis. In this way, it can be avoided the mechanisms associated with drug efflux pumps or the resistance to paclitaxel. (2)

One of these antineoplastic agents is ixabepilone for which an inverse relationship between its sensitivity and ER expression has been confirmed. A clinical trial conducted by Baselga and coworkers (24) on $26 \%$ TNBC women who were treated with 4 cycles of ixabepilone reported a pathological clinical response of $22 \%$ after completion the neoadjuvant chemotherapy compared to $46.1 \%$ in ER negative/HER2 positive, $10.6 \%$ in ER positive/ HER2 negative, and $20 \%$ in ER positive/HER2 positive which means that ixabepilone achieved relative good results in TNBC patients.

Other ongoing clinical trials are investigating the effect of the preoperative use of FEC (5-Fluorouracil - epirubicine - cyclophosphamide) and ixabepilone or compare paclitaxel with ixabepilone in the neoadjuvant setting of TNBC. $(2,13)$

\section{SURGERY IN THE NEOADJUVANT SETTING}

It is generally known that the diagnosis of a TNBC does not influence the choice between mastectomy and lumpectomy although it is accepted that TNBC are aggressive tumors with higher incidence in younger premenopausal women. It seems that decision between the two different surgical procedures is firstly based on clinicopathological variables and secondly on the patient decision. Furthermore, it has been demonstrated that a breast conservative surgery (BCS) in TNBCs presents a similar recurrence rate as other subtypes of $\mathrm{BCs}$ 
which means that sentinel lymph node (LN) dissection $->$ neoadjuvant chemotherapy $>$ BCS remains the appropriate solution in TNBC women. (25)

\section{CONCLUSIONS}

Although TNBC being sensitive to chemotherapy, early relapse is still frequent and the presence of distant metastases, especially brain metastases, indicate a very poor prognosis, as the chemosensitivity does not generally translate in an improvement of PFS or OS.

The efficacy of taxane-based regimens has been recognized hence they are recommended by the in- ternational and national guidelines as first-line treatment in TNBC in the neoadjuvant setting. The recommended first-line and second-line therapies are platinum-based regimens- cisplatin plus gemcitabine, carboplatin plus paclitaxel and carboplatin plus gemcitabine.

The second-line therapy consists of capecitabine or combination of capecitabine and vinorelbine, or gemcitabine and vinorelbine or a platinum-based regimen.

Other novel therapies which target the epidermal growth factor, fibroblastic growth factor or VEGF are currently under investigation.

\section{REFERENCES}

1. American Cancer Society. Cancer Facts and Figures 2010. Atlanta, Ga: American Cancer Society; 2010.

2. Wahba H.A., El-Hadaad H.A. Current approaches in treatment of triple-negative breast cancer. Cancer Biol Med 2015; 12:106-116.

3. Sorlie T., Tibshirani R., Parker J., et al. Repeated observation of breast tumor subtypes in independent gene expression data sets. Proc Natl Acad Sci USA. 2003; 100(14):8418-8423

4. Carey L.A., Perou C.M., Livasy C.A., et al. Race, breast cancer subtypes, and survival in the Carolina Breast Cancer Study. JAMA. 2006; 295(21): 2492-2502

5. Kaplan H.G., Malmgren J.A., Atwood M.K. Impact of triple negative phenotype on breast cancer prognosis. Poster presented at: 29th Annual San Antonio Breast Cancer Symposium; December 14-17, 2006; San Antonio, TX.

6. Mustacchi G., De Laurentiis M. The role of taxanes in triple-negative breast cancer: literature review. Drug Design, Development and Therapy 2015:9,4303-4318

7. Dent R.A., Mainwaring P.N., Tan T.J.Y., et al. Survival in triplenegative breast cancer (TNBC): Evidence from the SEER database 2010-2011. J Clin Oncol. 2015; 33(Suppl 15):e12075.

8. Chacón R.D., Costanzo M.V. Triple-negative breast cancer. Breast Cancer Res. 2010; 12 Suppl 2:S3.

9. Hudis C.A., Gianni L. Triple-negative breast cancer: an unmet medical need. Oncologist. 2011;16 Suppl 1:S1-S11

10. Gluz O., Liedtke C., Gottschalk N., Pusztai L., Nitz U., Harbeck N. Triple-negative breast cancer - current status and future directions. Ann Oncol 2009; 20:1913-1927.

11. Berrada N., Delaloge S., André F. Treatment of triple-negative metastatic breast cancer: toward individualized targeted treatments or chemosensitization? Ann Oncol 2010; 21 Suppl 7:vii30-viii35.

12. Geisler S., Lonning P.E., Aas T., Johnsen H., Fluge O., Haugen D.F., et al. Influence of TP53 gene alterations and c-erbB-2 expression on the response to treatment with doxorubicin in locally advanced breast cancer. Cancer Research 2001; 61:2505-2512.

13. Amos K.D., Adamo B., Anders C.K. Triple-negative breast cancer: an update on neoadjuvant clinical trials. Int J Breast Cancer 2012; 2012:385978.

14. Wang S., Yang H., Tong F., Zhang J., Yang D., Liu H., et al. Response to neoadjuvant therapy and disease free survival in patients with triple negative breast cancer. Gan To Kagaku Ryoho 2009; 36:255-258.

15. Rastogi P., Anderson S.J., Bear H.D., Geyer C.E., Kahlenberg M.S., Robidoux A., et al. Preoperative chemotherapy: updates of

National Surgical Adjuvant Breast and Bowel Project Protocols B-18 and B-27. J Clin Oncol 2008; 26: 778-785

16. Bhattacharyya A., Ear U.S., Koller B.H., Weichselbaum R.R., Bishop D.K. The breast cancer susceptibility gene BRCA1 is required for subnuclear assembly of Rad51 and survival following treatment with the DNA cross-linking agent cisplatin. J Biol Chem 2000; 275:23899-23903. 31

17. Garber J., Richardson A., Harris L., Mironn A., Silver D., Golshan M., et al. Neo-adjuvant cisplatin (CDDP) in triple-negative breast cancer (BC). Breast Cancer Res Treat 2006; 100:S149 (Abstr 3074).

18. Frasci G., Comella P., Rinaldo M., lodice G., Di Bonito M., D'Aiuto M., et al. Preoperative weekly cisplatin-epirubicinpaclitaxel with G-CSF support in triple-negative large operable breast cancer. Ann Oncol 2009; 20:1185-1192.

19. Zelnak A.B., Harichand-Herdt S., Styblo T.M., Rizzo M., Gabram S.G., Bumpers H.L., et al. Final results from randomized phase II trial of preoperative docetaxel (D) and capecitabine (C) given sequentially or concurrently for HER2-negative breast cancers. J Clin Oncol (Meeting Abstracts) 2011; 29:1118

20. Albain K.S., Allred D.C., Clark G.M. Breast cancer outcome and predictors of outcome: are there age differentials? J Natl Cancer Inst Monogr 1994; (16):35-42.

21. Gerber B., Loibl S., Eidtmann H., Rezai M., Fasching P.A., Tesch H., et al. Neoadjuvant bevacizumab and anthracycline-taxane-based chemotherapy in 678 triple-negative primary breast cancers: seconday endpoint analysis of the GeparQuinto study (GBG 44). Ann Oncol 2013; 24:2978-2984.

22. von Minckwitz G., Eidtmann H., Rezai M., Fasching P.A., Tesch H., Eggemann H., et al. Abstract S4-6: Neoadjuvant chemotherapy with or without Bevacizumab: primary efficacy endpoint analysis of the GEPARQUINTO Study (GBG 44). Cancer Res 2010; 70:S4-6.

23. Amos K.D., Adamo B., Anders C.K. Triple-negative breast cancer: an update on neoadjuvant clinical trials. Int J Breast Cancer, 2012:385978.

24. Baselga J., Zambetti M., Llombart-Cussac A., Manikhas G., Kubista E., Steger G.G., et al. Phase II genomics study of ixabepilone as neoadjuvant treatment for breast cancer. J Clin Oncol 2009; 27:526-534

25. Freedman G.M., Anderson P.R., Li T., Nicolaou N. Local-Regional Recurrence of Triple Negative Breast Cancer after Breast Conserving Surgery and Radiation. Cancer 2009; 115:946-951. 